\title{
The Effects of PCSK9 and Apolipoprotein E Functional Gene Variations On Hypercholesterolemia and Clinical Phenotype in Restenosis Patients After Percutaneous Coronary Angioplasty
}

\author{
Gulcin Ozkara \\ Istanbul University: Istanbul Universitesi \\ Zahra Javadova \\ Istanbul University: Istanbul Universitesi \\ Ezgi Aslan \\ Istanbul University: Istanbul Universitesi \\ A.Begum Ceviz \\ Istanbul University: Istanbul Universitesi \\ Gonca Candan \\ Istanbul University: Istanbul Universitesi \\ Ozgur Selim Ser \\ Istanbul University: Istanbul Universitesi \\ Onur Kilicarslan \\ Istanbul University: Istanbul Universitesi \\ Ozlem Kucukhuseyin \\ Istanbul University: Istanbul Universitesi \\ Cem Bostan \\ Istanbul University: Istanbul Universitesi \\ Ahmet Yildiz \\ Istanbul University: Istanbul Universitesi \\ Oguz Ozturk \\ Istanbul University: Istanbul Universitesi \\ Hulya Yilmaz Aydogan ( $\square$ yilmazh@istanbul.edu.tr) \\ Istanbul Universitesi https://orcid.org/0000-0002-8837-6664
}

\section{Research Article}

Keywords: PCSK9, ApoE, ApoER, mutation, restenosis, hypercholesterolemia

Posted Date: November 9th, 2021

DOI: https://doi.org/10.21203/rs.3.rs-1041248/v1

License: (c) (7) This work is licensed under a Creative Commons Attribution 4.0 International License. Read Full License 


\section{Abstract}

Background: In-stent-restenosis is a case restricting the benefits of percutaneous-transluminal coronary angioplasty (PTCA). PCSK9 controls LDLR levels, and variations in PCSK9, ApoE and ApoER genes may affect the development of restenosis. The aim of this study was to assess the effects of genetic variants on restenosis risk after PTCA.

Methods and Results: The study groups include 109 CAD-patients with restenosis (S-CAD) and 82 CAD-patients without restenosis (open-stent,OS-CAD). SNPs were analyzed by RT- PCR. PCSK9 levels were detected via ELISA method. The distributions of ApoE Epsilon, APOER (rs5174), PCSK9 rs2182833 and rs11206510 polymorphisms were found similar between study groups while the frequency of the PCSK9 E670G G allele in S-CAD group was found significantly higher than OS-CAD patients $(p=0.015)$. No difference was found between study groups in terms of the serum levels of PCSK9. LDL-C was found lower and HDL-C was found higher in OS-CAD group comparing with S-CAD group ( $p=0.042, p=0.008$, respectively). Frequencies of Type 2 DM and hyperlipidemia were also found higher in S-CAD group than OS-CAD group ( $p=0.007, p=0.001$, respectively) while EF\% was found lower in S-CAD group than OS-CAD group $(p=0.007)$.

Conclusions: Our findings indicate that although ApoE Epsilon, APOER (rs5174), PCSK9 rs2182833, rs11206510 and E670G polymorphisms has no effect on serum PCSK9 levels, PCSK9-rs505151G-allele and hyperlipidemia may be risk factors in the development of restenosis.

\section{Introduction}

The etiopathogenesis of coronary heart disease (CHD) involves many factors such as distorted blood pressure, lipoprotein, and glucose metabolism and hemostatic factors, which all lead to atherosclerosis and ischemia. Besides, these factors may also play a role in the response to treatment [1].

Atherosclerosis is a chronic inflammatory process affecting medium and large blood vessels in the cardiovascular system and characterized by atherogenic plaque formation $[2,3]$. Ischemia can be occurred mostly by atherosclerosis or by a blood clot or narrowing of the blood vessel, all of which block the blood flow to the heart muscle, and subsequently cause myocardial infarction (MI) [4, 5].

Percutaneous transluminal coronary angioplasty (PTCA), which provides rapid recovery in patients, is the basis of CHD treatment today. However, in $30-60 \%$ of the patients, ischemia can develop in the same myocardial region as the arteries recanalized after angioplasty re-narrow within six months [6]. Restenosis is defined as the narrowing of more than $50 \%$ of the vessel diameter recanalized after the PTCA procedure. It occurs as a result of the healing process in the vascular wall [7]. After the endothelial injury, blood flow is severely hampered at the injury site due to thrombosis formation as well as severe vasoconstriction and inflammatory response [8]. Since intra-stent restenosis development in many patients - especially those with diabetes and multiple heart and vascular disease - severely limits the benefits of the PTCA procedure, it is important to investigate the causes of restenosis in order to determine the optimal treatment.

Protein Convertase Subtilisin/ Kexin 9 (PCSK9) provides post-transcriptional regulation of low density lipoprotein receptor (LDLR) and thus the control of cellular LDLR levels. Gain and loss of function mutations in this gene are associated with hyper- and hypo-cholesterolemia [9-11]. PCSK9 binds to the LDLR located on the cell surface and triggers its destruction, thereby regulating the LDLR levels. LDL, which binds to LDLR, is taken into the cell with clathrin-coated vesicles, undergoes lysosomal degradation, and then returns to the finished receptor cell membrane. Increased LDLR function decreases plasma low density lipoprotein cholesterol (LDL-C) levels. Circulating PCSK9, a serine protease associated with hypercholesterolemia and CHD, also binds to the LDLR in the cell membrane, and together they enter the cell. It promotes the degradation of LDLR in the lysosome after it enters the cell, and thus the LDLR cannot be recycled to return to the cell membrane [12]. PCSK9 can also bind to LDLR intracellularly and trigger its destruction [13]. Because of these effects on LDLR levels and LDL-C levels, PCSK9 inhibitors have been used in the treatment of cholesterol-related diseases. These drugs have become an important drug group in recent years, especially in the treatment of LDL-C that cannot be reduced with statin therapy or for the patient group who cannot tolerate a statin. PCSK9, a subtilisinlike proprotein convertase family member with a chromosomal location of $1 \mathrm{p} 32.3$, binds to LDLR and provides lysosomal destruction of the receptor in the cell, and plays a role in cholesterol and fatty acid metabolism. PCSK9 increases plasma LDL levels by increasing LDLR degradation [14, 15]. Besides, it has been shown in experimental studies that PCSK9 plays a role in LDLR posttranscriptional regulation [16]. Therefore, PCSK9 functional gene variations have the potential to be a factor in the development of restenosis, as the variations in the PCSK9 gene that affect protein function affect LDLR and thus circulating LDL-C levels.

Another well-known ligand of LDLR is Apolipoprotein E (ApoE) which is a polymorphic glycoprotein that is the apoprotein component of LDL and very low density (VLDL) lipoproteins and serves as a ligand for chylomicron residue receptors [17]. It has been accepted that three major isoforms of $A p o E$ produce three alleles ( $\varepsilon 3, \varepsilon 2, \varepsilon 4$ ) at a single gene locus; Apo E3 (Cys 112 / Arg 158), Apo E2 (Cys 112 / Cys 158), and Apo E4 (Arg 112 / Arg 158) [18]. The most common allele is $\varepsilon 3$, therefore $A p o E 3$ is accepted as the main protein, whereas $A p o E 4$ and $A p o E 2$ are variants [19]. ApoE2 binding to the lipoprotein receptor is defective and is the most common form of $A p o E$ associated with type III hyperlipoproteinemia [20]. ApoE4 binds normally to the lipoprotein receptor, but this ApoE4 phenotype consists of higher plasma total and LDL-C levels (21). Most studies have found an association between CHD and the ApoE4 allele by its effects on cholesterol elevation. Moreover, ApoE4 has been shown to be an independent risk factor for CHD in the presence of other proposed mechanisms such as gene-gene and gene-environment interactions [22, 23]. On the other hand, there are also some studies showing that the effect of the $A p o E$ genotype on lipid metabolism may be modified by other genes. Pedersen et al. reported that gene-gene interactions between $L D L R$ and $A p o E$ loci contribute to the total- and LDL-C changes in the population [24]. In a study conducted by Yilmaz-Aydogan et al. gene-gene interaction between PPARD + 294T / C and ApoE epsilon polymorphisms was proposed and it was argued that this interaction could affect LDL-C levels in CHD patients [25]. Finally, it has been reported that the gene interaction between the PCSK9 gene E670G variation and the ApoE epsilon polymorphism affects plasma total- and LDL-C levels [26]. There are also studies showing that PCSK9 and ApoE proteins are involved in the process of atherogenesis via LDLR $[27,28]$. Therefore, functional variations in PCSK9 and ApoE genes should be examined in more detail in atherosclerosis-related diseases and lipid-lowering treatment protocols should be determined according to the presence of these genetic variations. 
By investigating the relationship between the formation of neo-atherosclerosis that develops in pathogenesis and the functional genetic variations that affect the benefit of treatment during the lipid-lowering treatment process in patients with PTCA and stenting, a different perspective will be provided to the clinical evaluation of the restenosis process and it will contribute to the determination of the values of $P C S K 9$ and ApoEvariations as genetic biomarkers. Within the scope of our study, target variations (R496W, E670G associated with gain of function and $Y 142 X$ variations associated with loss of function) in the PCSK 9 gene that affect circulating atherogenic LDL-C levels via LDLR in LDL metabolism, ApoE gene epsilon polymorphisms that affect ApoE's function and ApoE receptor $(A p o E R)$ polymorphism will be evaluated in terms of restenosis risk and lipid levels in patients with in-stent restenosis. Besides, the association of the serum PCSK9 levels with the genetic variations and the restenosis process will be examined.

\section{Materials And Methods Patient Selection}

Two age and gender-matched groups with a total of 191 cases who applied to Istanbul University, Institute of Cardiology were included in this study. All patients were diagnosed with coronary artery disease confirmed by coronary angiography and had drug-coated stent implantation at least six months ago. Study groups consist of patients with open stents (OS-CAD) and patients with stent restenosis (S-CAD). Patients with bare-metal stent (BMS) implantation, oncogenic patients, stent thrombosis, and patients not receiving regular antiplatelet therapy were not included in the study.

\section{Serum Lipid measurement}

Serum samples were obtained from the whole blood of overnight fasted participants. The enzymatic techniques were used to measure serum total cholesterol (Total-C), high density lipoprotein (HDL) cholesterol (HDL-C) and triglyceride (TG) levels. Serum LDL-C level was calculated using the Friedewald formula.

\section{Serum PCSK9 detection}

The PCSK9 protein was measured using the commercial ELISA kit (Biovendor, RD191473200R) in serum samples that were previously collected and stored at $-80^{\circ} \mathrm{C}$.

\section{Genotyping}

PureLink Genomic DNA Mini Kit (Thermo Fisher Scientific, K182002) was used for isolating genomic DNA from peripheral blood samples. Genotypes were determined by the Real-time PCR method using Roche LightCycler® 480 RT-PCR instrument. For RT-PCR analysis, TaqMan ${ }^{\circledR}$ (Suppl. Fig. 1) and LightSNIP® (Suppl. Fig. 2) primer-probe sets [PCSK9 rs374603772 (R496W) (ThermoFisher Scientific, C_357215641_10), rs505151 (E670G) (ThermoFisher Scientific, C_998744_10), rs67608943 (Y142X) (ThermoFisher Scientific, C_99339559_10), rs2182833 (ThermoFisher Scientific, C_2018193_10) and rs11206510 (ThermoFisher Scientific, C_32221221_10); ApoE gene epsilon variation rs429358 (ThermoFisher Scientific, C_3084793_20) and rs7412 (ThermoFisher Scientific, C_904973_10); and ApoER rs5174 (LightSNIP, Roche Diagnostic, Germany)], and two different master mixes [Jena Bioscience (PCR303) was used for TaqMan ${ }^{\circledR}$ probes, and LightCycler® FastStart DNA Master HybProbe (Roche Diagnostic, 03003248001) was used for LightSNIP® probes] were applied according to the manufacturer's instructions.

\section{Statistical Analysis}

The data were assessed using the SPSS 20 statistical analysis program. The distribution of the data was tested and then the parameters were compared between groups in accordance with the distribution. In order to compare differences between groups, the chi-square test for categorical variables, the Student's $t$ test and One-way analysis of variance (One-way Anova) for quantitative data were used in the case of the parameters that showed normal distribution. In the parameter types that do not show normal distribution, categorical variables were compared with the Mc-Nemar test and quantitative data were compared with Mann Whitney $U$ and Kruskal Wallis non-parametric tests. The associations between the genotype and allele distributions, serum PCSK9 levels and clinical symptoms and disorders, and the risks in disease development were determined by correlation and regression analysis.

Logistic regression analysis was performed to evaluate the effects of polymorphisms in the S-CAD group in the presence of cardiovascular risk factors. Multivariate logistic regression analysis was performed in the binary logistic regression model. Relative risk was determined by calculating the relative risk (OR, odds ratio) and confidence intervals $(\mathrm{Cl})$.

\section{Results}

The demographic, biochemical and clinical characteristics of the study groups are given in Table 1. Age and gender distributions were similar ( $p>0.05$ ). 
Table 1

Demographic, biochemical and clinical characteristics of the study groups

\begin{tabular}{|c|c|c|c|}
\hline & \multicolumn{2}{|l|}{ Groups } & \multirow[t]{3}{*}{ P-value } \\
\hline & \multirow[t]{2}{*}{ OS-CAD (n=82) } & \multirow{2}{*}{$\begin{array}{l}\text { S-CAD } \\
(n=109)\end{array}$} & \\
\hline & & & \\
\hline Age (Year) $(X \pm S D)$ & $62.33 \pm 10.29$ & $60.55 \pm 9.28$ & 0.192 \\
\hline Gender (Female/Male)(n) & $13 / 69$ & $21 / 88$ & 0.542 \\
\hline PCSK9 (ng/ml) & $456.50 \pm 227.29$ & $464.13 \pm 140.57$ & 0.767 \\
\hline Stent diameter $(X \pm S D)$ & $2.72 \pm 0.34$ & $2.72 \pm 0.28$ & 0.849 \\
\hline Stent lenght $(X \pm S D)$ & $22.15 \pm 4.78$ & $23.83 \pm 6.86$ & 0.064 \\
\hline Glucose $(\mathrm{mg} / \mathrm{dL})(\mathrm{X} \pm \mathrm{SD})$ & $123.77 \pm 51.93$ & $133.58 \pm 55.67$ & 0.147 \\
\hline HbA1c (\%)(X士 SD) & $6.28 \pm 1.14$ & $6.68 \pm 1.46$ & 0.015 \\
\hline Total-C (mg/dl) $(\mathrm{X} \pm \mathrm{SD})$ & $166.62 \pm 46.41$ & $170.39 \pm 47.93$ & 0.593 \\
\hline TG (mg/dl) $(X \pm S D)$ & $150.48 \pm 86.18$ & $175.25 \pm 121.12$ & 0.075 \\
\hline $\mathrm{HDL}-\mathrm{C}(\mathrm{mg} / \mathrm{dl})(\mathrm{X} \pm \mathrm{SD})$ & $46.37 \pm 13.53$ & $41.21 \pm 11.44$ & 0.008 \\
\hline LDL-C (mg/dl) $(X \pm S D)$ & $104.92 \pm 33.37$ & $116.94 \pm 43.47$ & 0.042 \\
\hline $\operatorname{VLDL}-\mathrm{C}(\mathrm{mg} / \mathrm{dl})(X \pm \mathrm{SD})$ & $28.22 \pm 12.81$ & $30.19 \pm 13.67$ & 0.315 \\
\hline ALT (U/L) & $19.76 \pm 8.36$ & $24.82 \pm 13.40$ & 0.032 \\
\hline AST (U/L) & $17.61 \pm 5.77$ & $20.76 \pm 8.21$ & 0.024 \\
\hline TSH (ulU/mL) & $1.73 \pm 1.03$ & $1.98 \pm 1.53$ & 0.216 \\
\hline Creatinine (mg/dL) & $0.94 \pm 0.29$ & $1.02 \pm 0.59$ & 0.186 \\
\hline BUN (mg/dL) & $17.39 \pm 9.82$ & $17.59 \pm 8.11$ & 0.869 \\
\hline $\mathrm{Na}(\mathrm{mEq} / \mathrm{L})$ & $139.19 \pm 14.59$ & $140.35 \pm 3.20$ & 0.339 \\
\hline $\mathrm{K}(\mathrm{mEq} / \mathrm{L})$ & $4.50 \pm 0.41$ & $4.61 \pm 0.42$ & 0.117 \\
\hline $\mathrm{Cl}(\mathrm{mmol} / \mathrm{L})$ & $101.62 \pm 3.11$ & $101.20 \pm 3.70$ & 0.446 \\
\hline WBC( $\mu \mathrm{L})$ & $9.60 \pm 1.31$ & $8.46 \pm 2.20$ & 0.283 \\
\hline $\mathrm{Hb}(\mathrm{g} / \mathrm{dL})$ & $13.54 \pm 2.20$ & $13.58 \pm 1.67$ & 0.878 \\
\hline Hct (\%) & $40.23 \pm 4.72$ & $40.15 \pm 4.85$ & 0.916 \\
\hline PIt $(\mu \mathrm{L})$ & $228.26 \pm 59.01$ & $243.78 \pm 69.10$ & 0.114 \\
\hline $\mathrm{EF}(\%)$ & $54.61 \pm 8.72$ & $50.89 \pm 11.46$ & 0.007 \\
\hline Hypertension (\%) & 49.4 & 61.3 & 0.105 \\
\hline Type 2 DM (\%) & 39.2 & 59.4 & 0.007 \\
\hline Hyperlipidemia (\%) & 34.2 & 66.0 & 0.001 \\
\hline $\begin{array}{l}\text { OS-CAD; Open stent corona } \\
\text { Triglyceride, HDL-C; HDL-ch } \\
\text { thyroid stimulating hormor } \\
\text { platelet, EF; ejection fractio }\end{array}$ & $\begin{array}{l}\text {-CAD; Coronary } \\
\text { I, VLDL-C; VLDL-c } \\
\text { sodium, K; potas } \\
\text { ellitus }\end{array}$ & $\begin{array}{l}\text { t group with stent } \\
\text { in transaminase; } \\
3 C \text {; white blood ce }\end{array}$ & $\begin{array}{l}\text { otal-cholesterol, TG } \\
\text { minase, TSH; } \\
\text { Hct; hematocrit, Plt; }\end{array}$ \\
\hline
\end{tabular}

$\operatorname{HbA1c}(p=0.015), \operatorname{LDL}-\mathrm{C}(p=0.042)$ levels were higher, and HDL-C $(p=0.008)$ and EF $(p=0.007)$ were found lower in the S-CAD group compared to the OS-CAD patients (Table 1). Frequencies of Type 2 DM and hyperlipidemia were also found higher in S-CAD group than 0S-CAD group ( $p=0.007, p=0.001$, respectively)

Table 2 shows the genotype and allele distribution of ApoE epsilon and ApoE Receptor (ApoER) rs5174 polymorphisms, and PCSK9 E670G (rs505151), rs2182833 and rs11206510 mutations. Interestingly, when PCSK9 R496W (rs374603772) and Y142X (rs67608943) mutations were evaluated, the ancestral C allele was found in the study groups and all genotypes were identified as CC (S-CAD: 109, OS-CAD: 82). The frequency of the E670G G allele in S-CAD group was significantly higher than OS-CAD patients $(p=0.015)$. Other genotype and allele frequencies were found similar between study groups $(p>0.05)$. 
Distribution of $A p o E, A p o E R$ and $P C S K 9$ gene variations in study groups *

\begin{tabular}{|c|c|c|c|}
\hline \multirow{2}{*}{$\begin{array}{l}\text { Gene variations } \\
\text { Apo E Epsilon Polymorphism }\end{array}$} & \multirow[b]{2}{*}{ Apo E Genotypes } & \multicolumn{2}{|l|}{ Groups } \\
\hline & & OS-CAD $(n=82)$ & S-CAD $(n=109)$ \\
\hline & E2E2 & - & - \\
\hline & E2E3 & $9(11.0 \%)$ & $14(12.8 \%)$ \\
\hline & E2E4 & $1(1.2 \%)$ & - \\
\hline & E3E3 & $63(76.8 \%)$ & $77(70.6 \%)$ \\
\hline & E3E4 & $8(9.8 \%)$ & $17(15.6 \%)$ \\
\hline & E4E4 & $1(1.2 \%)$ & $1(0.9 \%)$ \\
\hline & \multicolumn{3}{|l|}{ Apo E Alleles } \\
\hline & E2 & $10(6.09 \%)$ & $14(6.42 \%)$ \\
\hline & E3 & $143(87.20 \%)$ & $179(82.11 \%)$ \\
\hline & E4 & $11(6.71 \%)$ & $19(8.72 \%)$ \\
\hline \multirow[t]{7}{*}{ ApoER-rs5174 polymorphism } & ApoER (rs5174) Genotypes & $(n=82)$ & $(n=108)$ \\
\hline & GG & $37(45.1 \%)$ & $54(50.0 \%)$ \\
\hline & AA & $6(7.3 \%)$ & $17(15.7 \%)$ \\
\hline & GA & $39(47.6 \%)$ & $37(34.3 \%)$ \\
\hline & \multicolumn{3}{|l|}{ ApoER Alleles } \\
\hline & G & $113(68.90 \%)$ & $145(67.13 \%)$ \\
\hline & A & $51(31.10 \%)$ & $71(32.87 \%)$ \\
\hline \multirow[t]{7}{*}{ PCSK9-rs505151 (E670G) mutation } & PCSK9-rs505151(E670G) Genotypes & $(n=82)$ & $(n=109)$ \\
\hline & AA & $55(67.1 \%)$ & $54(49.5 \%)$ \\
\hline & GG & $5(6.1 \%)$ & $6(5.5 \%)$ \\
\hline & AG & $22(26.8 \%)$ & $49(45.0 \%)$ \\
\hline & \multicolumn{3}{|l|}{ PCSK9- rs505151(E670G) Alleles } \\
\hline & A & $132(80.49 \%)$ & $157(72.02 \%)$ \\
\hline & G & $32(19.51 \%)$ & $61(27.98 \%) * \star$ \\
\hline \multirow[t]{7}{*}{ PCSK9-rs2182833 mutation } & PCSK9-rs2182833 Genotypes & $(n=82)$ & $(n=109)$ \\
\hline & AA & $51(62.2 \%)$ & $63(57.8 \%)$ \\
\hline & GG & $10(12.2 \%)$ & $7(6.4 \%)$ \\
\hline & AG & $21(25.6 \%)$ & $39(35.8 \%)$ \\
\hline & \multicolumn{3}{|l|}{ PCSK9-rs2182833 Alleles } \\
\hline & A & $123(75.0 \%)$ & $165(75.69 \%)$ \\
\hline & G & $41(25.0 \%)$ & $53(24.31 \%)$ \\
\hline \multirow[t]{7}{*}{ PCSK9-rs11206510 mutation } & PCSK9-rs11206510 Genotypes & $(n=82)$ & $(n=109)$ \\
\hline & TT & $62(75.6 \%)$ & $84(77.1 \%)$ \\
\hline & $\mathrm{cc}$ & $2(2.4 \%)$ & $2(1.8 \%)$ \\
\hline & TC & $18(22.0 \%)$ & $23(21.1 \%)$ \\
\hline & \multicolumn{3}{|l|}{ PCSK9 rs11206510 Alleles } \\
\hline & $\mathrm{T}$ & $142(86.59 \%)$ & $191(87.61 \%)$ \\
\hline & C & $22(13.41 \%)$ & $27(12.39 \%)$ \\
\hline \multicolumn{4}{|c|}{$\begin{array}{l}\text { *, PCSK9 rs67608943 (Y142X) and rs374603772 (R496W) mutations were not detected in the study groups. Only ancestral alleles were observed in a } \\
\text { groups. }\end{array}$} \\
\hline
\end{tabular}


The binary multivariate logistic regression analysis was used to analyze the effects of risk factors determined in univariate statistical analysis in the development of restenosis and CAD in the presence of other variables (Table 3). As a result of this analysis, hyperlipidemia ( $p<0.001)$ and the PCSK9 E670G (rs505151) G allele ( $p=0.02)$ were determined for the risk of restenosis between S-CAD and OS-CAD groups.

Table 3

Binary - multivariate logistic regression analysis in the study groups

\begin{tabular}{llllll} 
Model & Dependent Variable & Independent Variable & Exp(B) (GR) & $\boldsymbol{P}$ & $95 \% \mathrm{cl}$ \\
S-CAD & S-CAD & Type 2 DM & 1.661 & 0.135 \\
vs & & Hyperlipidemia & 3.116 & $<.853-3.234$ \\
OS-CAD & & PCSK9 rs505151 G allele & 2.135 & 0.001 & $1.607-6.042$ \\
\hline
\end{tabular}

In binary - multivariate logistic regression analysis, risk factors for the S-CAD and OS-CAD were determined in the presence of other variables by using the significant parameters from univariate statistical analysis. S-CAD; Coronary artery disease patients with stent restenosis, OS-CAD; Open-stent coronary artery disease patient group, Type 2 DM; Type 2 diabetes mellitus.

Table 4 shows the comparison of biochemical characteristics with genotypes in OS-CAD group. Higher serum level of VLDL-C was observed in OS-CAD patients carrying E2 allele $(\mathrm{p}=0.022)$. In addition, although the difference was not significant, serum level of triglyceride was higher than those with not carrying $E 2$ allele ( $181.20 \pm 95.17$ vs $146.03 \pm 84.62$, respectively, p>0.05). On the other hand, ApoE3 and ApoE4 alleles were not found to be associated with serum lipid levels in S-CAD patients, however, the only significance was seen in median age $(p=0.022)$ for $A p o E 3$ and stent length ( $p=0.002)$ for $A p o E 4$. A significance was found between PCSK-rs505151 [E670G (A>G)] mutation and serum triglyceride levels $(\mathrm{p}=0.05)$. On the other hand, patients carrying $A p o E R$ rs5174 [G>A (C> T)] G allele (GG+GA genotypes) had higher levels of glycose than those with homozygous $A$ allele (AA genotype) (p< 0.001$)$. The stent diameter is greater in ApoER-rs5174 A allele (AA+GA genotypes) carriers than GG genotype carriers ( $\mathrm{p}=0.024)$, while it was lower in $P C S K 9$-rs2182833 [A> G] rare $\mathrm{G}$ allele (GG+GA genotype) carriers than those with AA genotype $(\mathrm{p}=0.024)$. No association was found between $P C S K 9$-rs $11206510[T>C]$ polymorphism and biochemical parameters ( $p>0.05)$ (Table 4). 
Table 4

Comparison of biochemical characteristics with genotypes in the OS-CAD group

\begin{tabular}{|c|c|c|c|c|c|c|c|c|c|c|c|c|c|}
\hline \multirow{2}{*}{$\begin{array}{l}\text { OS-CAD } \\
\text { Group }\end{array}$} & \multicolumn{2}{|c|}{ ApoE2 Allele } & \multicolumn{2}{|c|}{ ApoE3 Allele } & \multicolumn{2}{|c|}{ ApoE4 Allele } & \multicolumn{2}{|c|}{$\begin{array}{l}\text { PCSK9 E670G A>G } \\
\text { (rs505151) }\end{array}$} & \multicolumn{2}{|c|}{$\begin{array}{l}\text { ApoER (rs5174) } \\
\text { G>A G Allele }\end{array}$} & \multicolumn{2}{|c|}{$\begin{array}{l}\text { ApoER (rs5174) } \\
\text { G>A A Allele }\end{array}$} & \multirow{2}{*}{$\begin{array}{l}\begin{array}{l}P C S A \\
A>G\end{array} \\
\begin{array}{c}G A+C \\
(n=2\end{array}\end{array}$} \\
\hline & $\begin{array}{l}\text { ApoE2+ } \\
(n=10)\end{array}$ & $\begin{array}{l}\text { ApoE2- } \\
(n=69)\end{array}$ & $\begin{array}{l}\text { ApoE3+ } \\
(n=78)\end{array}$ & $\begin{array}{l}\text { ApoE3 } \\
(n=1)\end{array}$ & $\begin{array}{l}\text { ApoE4+ } \\
(n=10)\end{array}$ & $\begin{array}{l}\text { ApoE4- } \\
(n=69)\end{array}$ & $\begin{array}{l}\text { GG+GA } \\
(n=27)\end{array}$ & $\begin{array}{l}\text { AA } \\
(n=52)\end{array}$ & $\begin{array}{l}\text { GG+GA } \\
(n=75)\end{array}$ & $\begin{array}{l}\text { AA } \\
(n=4)\end{array}$ & $\begin{array}{l}\mathrm{GA}+\mathrm{AA} \\
(\mathrm{n}=43)\end{array}$ & $\begin{array}{l}\text { GG } \\
(n=36)\end{array}$ & \\
\hline \multirow[t]{2}{*}{ Age (year) } & 61.50 & 62.45 & 62.63 & \multirow[t]{2}{*}{39.00} & 57.20 & 63.07 & 61.33 & 62.85 & 62.24 & 64.00 & 63.88 & 60.47 & $59.3 \varepsilon$ \\
\hline & \pm 10.05 & \pm 10.39 & $\pm 10.00 *$ & & \pm 11.67 & \pm 9.95 & \pm 10.00 & \pm 10.49 & \pm 10.53 & \pm 3.16 & \pm 11.68 & \pm 8.10 & \pm 9.14 \\
\hline \multirow{2}{*}{$\begin{array}{l}\text { Glucose } \\
\text { (mg/dL) }\end{array}$} & 120.20 & 124.29 & 124.14 & \multirow[t]{2}{*}{95.00} & 109.20 & 125.88 & 116.48 & 127.55 & 125.26 & 95.75 & 114.72 & 134.58 & $129 . €$ \\
\hline & \pm 46.14 & \pm 53.00 & \pm 52.16 & & \pm 46.75 & \pm 52.61 & \pm 49.59 & \pm 53.17 & \pm 52.87 & \pm 6.07 & \pm 42.75 & \pm 59.96 & \pm 52.6 \\
\hline \multirow[t]{2}{*}{$\mathrm{HbA1C}$} & 6.71 & 6.21 & 6.29 & \multirow[t]{2}{*}{5.40} & 6.30 & 6.27 & 6.19 & 6.32 & 6.30 & 5.77 & 6.04 & 6.55 & 6.44 \\
\hline & \pm 1.86 & \pm 1.00 & \pm 1.14 & & \pm 1.83 & \pm 1.02 & \pm 1.19 & \pm 1.12 & \pm 1.16 & \pm 0.45 & \pm 0.77 & \pm 1.43 & $\pm 1.3 \mathrm{C}$ \\
\hline \multirow{2}{*}{$\begin{array}{l}\text { Stent } \\
\text { Diameter }\end{array}$} & 2.82 & 2.69 & 2.71 & \multirow[t]{2}{*}{2.50} & 2.62 & 27 & 271 & 2.71 & 2.72 & 2.56 & 2.79 & 2.62 & 2.60 \\
\hline & \pm 0.51 & \pm 0.30 & \pm 0.33 & & \pm 0.17 & \pm 0.35 & \pm 0.31 & \pm 0.35 & \pm 0.34 & \pm 0.12 & $\pm 0.37 \rrbracket$ & \pm 0.25 & $\pm 0.2 \mathrm{C}$ \\
\hline \multirow{2}{*}{$\begin{array}{l}\text { Stent } \\
\text { Lenght }\end{array}$} & 22.50 & 22.10 & 22.15 & \multirow[t]{2}{*}{22.00} & 26.40 & 21.53 & 22. & 22.03 & 22 & 21.50 & 22.72 & 1.47 & 2.06 \\
\hline & \pm 5.87 & \pm 4.65 & \pm 4.81 & & $\pm 5.23 ¥$ & \pm 4.42 & \pm 5.04 & \pm 4.68 & \pm 4.85 & \pm 3.41 & \pm 5.03 & \pm 4.43 & \pm 4.55 \\
\hline \multirow{2}{*}{$\begin{array}{l}\text { Total-C } \\
(\mathrm{mmol} / \mathrm{L})\end{array}$} & 159.20 & 167.70 & 167.30 & \multirow[t]{2}{*}{130.00} & 161.90 & 167.30 & 164.63 & 167.65 & 165.21 & 193.00 & 163.58 & 170.25 & 169.C \\
\hline & \pm 41.16 & \pm 47.30 & \pm 48.59 & & \pm 28.31 & \pm 48.58 & \pm 40.49 & \pm 99.35 & \pm 46.22 & \pm 48.25 & \pm 40.34 & \pm 53.12 & \pm 38.3 \\
\hline \multirow{2}{*}{$\begin{array}{l}\text { TG } \\
(\mathrm{mmol} / \mathrm{L})\end{array}$} & 181.20 & 146.03 & 151.38 & \multirow[t]{2}{*}{80.00} & 120.10 & 154.88 & 128.96 & 161.65 & 149.60 & 167.00 & 150.23 & 150.78 & 169. \\
\hline & \pm 95.17 & \pm 84.62 & \pm 86.36 & & \pm 64.36 & \pm 88.40 & \pm 46.80 & $\stackrel{ \pm 99.34}{£}$ & \pm 87.82 & \pm 49.64 & \pm 83.42 & \pm 90.54 & \pm 97.5 \\
\hline \multirow{2}{*}{$\begin{array}{l}\text { HDL-C } \\
\text { (mmol/L) }\end{array}$} & 45.00 & 46.56 & 46.44 & \multirow[t]{2}{*}{40.00} & 48.90 & 46.00 & 46.48 & 46.30 & 45.94 & 54.25 & 47.83 & 44.61 & $46.0 \mathrm{C}$ \\
\hline & \pm 10.97 & \pm 13.91 & \pm 13.59 & & \pm 20.59 & \pm 12.35 & \pm 14.42 & \pm 13.18 & \pm 13.50 & \pm 13.07 & \pm 15.06 & \pm 11.38 & \pm 13.6 \\
\hline \multirow{2}{*}{$\begin{array}{l}\text { LDL-C } \\
(\mathrm{mmol} / \mathrm{L})\end{array}$} & 95.30 & 106.31 & 105.02 & \multirow[t]{2}{*}{100.00} & 104.20 & 105.03 & 110.00 & 102.29 & 103.80 & 126.00 & 104.83 & 105.03 & 109.9 \\
\hline & \pm 31.18 & \pm 33.65 & \pm 35.00 & & \pm 19.73 & \pm 35.00 & \pm 36.81 & \pm 31.48 & \pm 32.63 & \pm 45.40 & \pm 32.22 & \pm 35.13 & $\pm 33 . \mathrm{C}$ \\
\hline \multirow{2}{*}{$\begin{array}{l}\text { VLDL-C } \\
(\mathrm{mmol} / \mathrm{L})\end{array}$} & 36.24 & 27.02 & 28.38 & \multirow[t]{2}{*}{16.00} & 24.02 & 28.85 & 25.79 & 29.53 & 27.93 & 33.40 & 28.23 & 28.20 & 31.67 \\
\hline & $\pm 19.03^{*}$ & \pm 11.33 & \pm 12.81 & & \pm 12.87 & \pm 12.78 & \pm 9.36 & \pm 14.25 & \pm 12.94 & \pm 9.92 & \pm 11.88 & \pm 14.02 & \pm 15.3 \\
\hline PCSK9 & 436.00 & 459.55 & 453.95 & \multirow[t]{2}{*}{649.89} & 531.07 & 446.62 & 493.40 & 436.56 & 453.01 & 497.70 & 478.52 & 427.12 & 490.7 \\
\hline (ng/ml) & \pm 138.45 & \pm 238.33 & \pm 227.69 & & \pm 362.86 & \pm 205.02 & \pm 257.53 & \pm 209.24 & \pm 235.30 & \pm 89.09 & \pm 222.52 & \pm 233.67 & \pm 276 \\
\hline
\end{tabular}

Results are given as mean \pm standard deviation. Total-C; total-cholesterol, TG; Triglyceride, HDL-C; HDL-cholesterol, LDL-C; LDL-cholesterol, VLDL-C; VLDL-cho $p=0.05 ; \$$. $p<0.001 ; \otimes . p=0.024$

In Table 5, the distributions of the biochemical parameters among genotypes in the S-CAD group were shown. ApoE epsilon polymorphism E2 allele was not found to be associated with serum lipid levels in S-CAD patients. However, similar to OS-CAD group, S-CAD patients carrying E2 allele had higher serum levels of triglyceride $(223.07 \pm 169.38$ vs. $167.97 \pm 11.46, p>0.05)$. A significant difference was observed in glucose and HbA1c levels between the groups with and without the $E 3$ allele ( $p<0.001$ and $p=0.044$, respectively). On the other hand, higher level of triglyceride was observed in $E 4$ allele carriers ( $p<0.001$ ). Regarding PCSK9-E670G A> G polymorphism, G allele (GG+GA genotypes) was found to be associated with low fasting blood glucose level ( $p=0.019)$ and stent diameter $(\mathrm{p}<0.001)$. Besides, S-CAD patients carrying the $E 670 G \mathrm{G}$ allele also had higher LDL-C levels compared to the AA genotype carriers ( $\mathrm{p}=0.036)$. PCSK9-rs11206510 T>C polymorphism rare $\mathrm{C}$ allele (CC+CT genotypes) was observed to be associated with lower HbA1c levels compared to the TT genotype $(p=0.021)$. No significant effect of PCSK9-rs2182833 A>G polymorphism on biochemical parameters was observed in the S-CAD group ( $p>0.05)(T a b l e ~ 5)$. 
Table 5

Comparison of biochemical characteristics with genotypes in the S-CAD group

\begin{tabular}{|c|c|c|c|c|c|c|c|c|c|c|c|c|}
\hline \multirow{2}{*}{$\begin{array}{l}S-C A D \\
\text { group } \\
\text { Parameter }\end{array}$} & \multicolumn{2}{|c|}{ ApoE2 Allele } & \multicolumn{2}{|c|}{ ApoE3 Allele } & \multicolumn{2}{|c|}{ ApoE4 Allele } & \multicolumn{2}{|c|}{$\begin{array}{l}\text { PCSK9 E670G A>G } \\
\text { (rs505151) }\end{array}$} & \multicolumn{2}{|c|}{$\begin{array}{l}\text { ApoER (rs5174) } \\
\text { G>A G Allele }\end{array}$} & \multicolumn{2}{|c|}{$\begin{array}{l}\text { ApoER (rs5174) } \\
\text { G>A A Allele }\end{array}$} \\
\hline & $\begin{array}{l}\text { ApoE2+ } \\
(n=14)\end{array}$ & $\begin{array}{l}\text { ApoE2- } \\
(n=92)\end{array}$ & $\begin{array}{l}\text { ApoE3+ } \\
(n= \\
105)\end{array}$ & $\begin{array}{l}\text { ApoE3- } \\
(n=1)\end{array}$ & $\begin{array}{l}\text { ApoE4+ } \\
(n=18)\end{array}$ & $\begin{array}{l}\text { ApoE4- } \\
(n=88)\end{array}$ & $\begin{array}{l}\text { GG+GA } \\
(n=55)\end{array}$ & $\begin{array}{l}\text { AA } \\
(n=51)\end{array}$ & $\begin{array}{l}\text { GG+GA } \\
(n=88)\end{array}$ & $\begin{array}{l}\text { AA } \\
(n=17)\end{array}$ & $\begin{array}{l}\text { GA+AA } \\
(n=51)\end{array}$ & $\begin{array}{l}\text { GG } \\
(n=54)\end{array}$ \\
\hline \multirow[t]{2}{*}{ Age (year) } & 60.21 & 60.60 & 60.62 & 53.00 & 58.28 & 61.01 & 61.58 & 59.43 & 61.22 & 56.94 & 59.22 & 61.76 \\
\hline & \pm 7.11 & \pm 9.60 & \pm 9.29 & & \pm 7.46 & \pm 9.58 & \pm 9.71 & \pm 8.75 & \pm 9.22 & \pm 9.28 & \pm 9.65 & \pm 8.91 \\
\hline \multirow{2}{*}{$\begin{array}{l}\text { Glucose } \\
\text { (mg/dL) }\end{array}$} & 133.71 & 133.55 & 131.21 & \multirow[t]{2}{*}{$381.00 *$} & 138.88 & 132.48 & 121.45 & \multirow{2}{*}{$\begin{array}{l}146.647 \pm 57.74 \\
£\end{array}$} & 133.83 & 134.17 & 142.82 & 125.44 \\
\hline & \pm 40.49 & \pm 57.81 & \pm 50.35 & & \pm 69.14 & \pm 52.91 & \pm 51.27 & & \pm 57.31 & \pm 49.11 & \pm 62.50 & \pm 47.80 \\
\hline \multirow[t]{2}{*}{$\mathrm{HbA1C}$} & 6.62 & 6.68 & 6.65 & \multirow[t]{2}{*}{$9.60 ¥}$. & 6.79 & 6.65 & 6.47 & 6.89 & 6.73 & 6.44 & 6.86 & 6.52 \\
\hline & \pm 1.66 & \pm 1.43 & \pm 1.44 & & \pm 1.14 & \pm 1.52 & \pm 1.39 & \pm 1.51 & \pm 1.51 & \pm 1.18 & \pm 1.56 & \pm 1.35 \\
\hline \multirow{2}{*}{$\begin{array}{l}\text { Stent } \\
\text { Diameter }\end{array}$} & 2.67 & 2.73 & 2.72 & \multirow[t]{2}{*}{2.75} & 2.79 & 2.71 & 2.62 & 2.83 & 2.72 & 2.70 & 2.69 & 2.75 \\
\hline & \pm 0.30 & \pm 0.27 & \pm 0.27 & & \pm 0.26 & \pm 0.27 & \pm 0.22 & $\pm 0.28^{\star}$ & \pm 0.29 & \pm 0.18 & \pm 0.26 & \pm 0.28 \\
\hline \multirow{2}{*}{$\begin{array}{l}\text { Stent } \\
\text { Lenght }\end{array}$} & 23.42 & 23.89 & 23.82 & \multirow[t]{2}{*}{24.00} & 23.61 & 23.87 & 23.45 & 24.23 & 23.94 & 23.23 & 24.37 & 23.31 \\
\hline & \pm 6.81 & \pm 6.89 & \pm 6.88 & & \pm 4.91 & \pm 7.21 & \pm 6.82 & \pm 6.93 & \pm 7.13 & \pm 5.60 & \pm 7.88 & \pm 5.82 \\
\hline \multirow{2}{*}{$\begin{array}{l}\text { Total-C } \\
(\mathrm{mmol} / \mathrm{L})\end{array}$} & 162.78 & 171.54 & 169.64 & \multirow[t]{2}{*}{248.00} & 168.00 & 170.87 & 177.76 & 162.43 & 169.30 & 178.29 & 168.39 & 173.00 \\
\hline & \pm 52.48 & \pm 47.40 & \pm 47.55 & & \pm 48.17 & \pm 48.14 & \pm 53.00 & \pm 40.81 & \pm 48.60 & \pm 45.42 & \pm 43.19 & \pm 52.45 \\
\hline \multirow{2}{*}{$\begin{array}{l}\text { TG } \\
(\mathrm{mmol} / \mathrm{L})\end{array}$} & 223.07 & 167.97 & 170.73 & \multirow[t]{2}{*}{649.00} & 187.50 & 172.74 & 162.44 & 189.06 & 170.27 & 196.94 & 163.41 & 185.15 \\
\hline & \pm 169.38 & \pm 111.46 & \pm 112.39 & & $\pm 148.05^{\star}$ & \pm 115.69 & \pm 109.90 & \pm 131.86 & \pm 117.80 & \pm 141.02 & \pm 108.55 & \pm 132.7 \\
\hline \multirow{2}{*}{$\begin{array}{l}\text { HDL-C } \\
(\mathrm{mmol} / \mathrm{L})\end{array}$} & 37.92 & 41.71 & 41.29 & \multirow[t]{2}{*}{33.00} & 40.27 & 41.40 & 42.40 & 39.94 & 41.38 & 41.29 & 41.37 & 41.37 \\
\hline & \pm 7.72 & \pm 11.86 & \pm 11.47 & & \pm 8.90 & \pm 11.93 & \pm 12.14 & \pm 10.61 & \pm 11.75 & \pm 9.57 & \pm 10.56 & \pm 12.21 \\
\hline \multirow{2}{*}{$\begin{array}{l}\text { LDL-C } \\
(\mathrm{mmol} / \mathrm{L})\end{array}$} & 99.00 & 119.67 & 116.47 & \multirow[t]{2}{*}{166.00} & 115.55 & 117.22 & 125.32 & 107.90 & 116.34 & 122.47 & 113.78 & 120.68 \\
\hline & \pm 40.41 & \pm 43.47 & \pm 43.41 & & \pm 44.13 & \pm 43.58 & $\stackrel{ \pm}{\$}$ & \pm 34.18 & \pm 44.27 & \pm 40.05 & \pm 37.80 & \pm 48.36 \\
\hline \multirow{2}{*}{$\begin{array}{l}\text { VLDL-C } \\
(\mathrm{mmol} / \mathrm{L})\end{array}$} & 33.11 & 29.78 & 30.18 & \multirow[t]{2}{*}{-} & 28.22 & 30.55 & 29.07 & 31.44 & 29.30 & 33.60 & 28.99 & 30.98 \\
\hline & \pm 17.69 & \pm 13.10 & \pm 13.67 & & \pm 11.14 & \pm 14.12 & \pm 13.10 & \pm 14.32 & \pm 13.20 & \pm 15.52 & \pm 11.57 & \pm 15.40 \\
\hline PCSK9 & 489.74 & 460.67 & 466.63 & 214.10 & 443.72 & 468.55 & 446.54 & 481.37 & 459.85 & 485.96 & 455.74 & 472.18 \\
\hline (ng/ml) & \pm 145.89 & \pm 140.33 & \pm 139.00 & & \pm 162.16 & \pm 136.14 & \pm 122.28 & \pm 155.74 & \pm 126.92 & \pm 200.82 & \pm 134.77 & \pm 147.8 \\
\hline
\end{tabular}

Results are given as mean \pm standard deviation. Total-C; total-cholesterol, TG; Triglyceride, HDL-C; HDL-cholesterol, LDL-C; LDL-cholesterol, VLDL-C; VLDL-cho $p=0.036 ;$; $p=0.021$

\section{Discussion}

PCSK9 increases plasma LDL levels by increasing LDLR degradation $[14,15]$. Gain of function mutations in the PCSK9 gene may cause an increase in plasma LDL-C and CHD risk by decreasing LDLR levels in the liver [9], while loss of function mutations may cause an increase in LDLR levels and a decrease in plasma LDL-C levels and protect against CHD formation [29, 30]. In our study, gain of function mutations of PCSK9 gene E670G (rs505151), R496W (rs374603772), and the loss of function mutation Y142X (rs6768943) which were all thought to affect serum LDL-C levels and PCSK9 rs11206510 polymorphism [31, 32] and rs2182833 [33] which have been associated with LDL-C levels previously and latter have been shown to possess no effect on PCSK9 function were examined to show the effects of variations on serum lipid and PCSK9 levels and restenosis risk in CHD patients.

According to RT-PCR results of the screening for $R 496 W$ and $Y 142 X$ mutations, all of the subjects in our study groups (191 people in total) had CC genotype, and no mutant allele was observed. In the study of Eroglu et al. which was performed in Aegean region of Turkey, the genotype distributions of $P C S K 9$ R496W mutation were reported as CT $6 \%$, TT $0.5 \%$ in dyslipidemia group $(n=200), C T 0.5 \%$, TT $0 \%$ in the control group ( $n=201)$. They also reported that total-C ( $=$ 0.021), TG ( $\mathrm{p}=0.0001), \mathrm{HDL}-\mathrm{C}(\mathrm{p}=0.028)$, and LDL-C ( $\mathrm{p}=0.028)$ levels were higher in individuals with mutations of PCSK9-rs374603772 (R496W) and rs137852912 (D374Y) than those without these mutations [34]. Kaya et al. conducted a study with 80 patients with familial hypercholesterolemia, and $R 496 W$ mutation was observed in seven people (8.7\%) and one of them was found to be homozygous. In this study, no difference was found in terms of cardiovascular disease risk between the patient group with any of the gain-of-function mutations ( F216L, R496W, S127R, and D374Y) in the PCSK9 gene and 
individuals without the mutation [35]. Cohen et al. reported low LDL-C plasma levels in 128 individuals with loss of function mutations ( $Y 142 X$ and C679X) [29].

In our study, although statistical significance could not be obtained in S- and OS-CAD groups, it was observed that LDL-C levels were higher in G allele carriers than those who did not. In the study of Postmus et al., the variation of rs2182833 was found to be associated with LDL-C levels in elderly individuals (70-82 years of age) with vascular disease or at risk of vascular disease $(p<0.01)$. Moreover, LDL-C levels were observed as high to low homozygous> heterozygous, respectively [33]. In a study which gene-environment interaction in cardiovascular diseases was assessed with the STANISLAS cohort consisting of $\sim 861$ people who were followed up for 18-23 years, no relationship was observed between PCSK9-rs2182833 and rs11206510 variations and LDL-C and PCSK9 levels [36]. Similarly, in our study, no relationship was found between the PCSK9-rs11206510 polymorphism and LDL-C and PCSK9 levels. Genotype and allele frequencies of this polymorphism were similar among our study groups $(p<0.05)$. Similar to our findings, the rs 11206510 polymorphism was not associated with CHD in the Asian population in a meta-analysis study conducted on 813 (290 CHD, 193 non-CHD patients, and 330 healthy controls) subjects [37]. In another study conducted in 1880 individuals in the Italian population that investigated PCSK 9 gene variations and LDL-C levels and the risk of myocardial infarction (MI), rs2182833 was not associated with LDL-C, total-C and MI risk, while rs11206510 minor allele (C) was associated with with low levels of LDL-C $(\mathrm{OR}=0.82,95 \% \mathrm{Cl}=0.73-0.93, \mathrm{p}=1.89 \times 10-3)$ and total-C $(\mathrm{OR}=0.80,95 \% \mathrm{Cl}=0.72-0.89, \mathrm{p}=8.12 \times 10-5)$ but not with the risk of $\mathrm{MI}[32]$. In the study of Qi et al., the rs11206510 polymorphism was found to be associated with CHD in diabetic patients [38].

In our study, the PCSK9-rs11206510 T>C polymorphism in the S-CAD group was observed to be associated with lower HbA1c levels compared to the rare $\mathrm{C}$ allele TT genotype $(p=0.021)$. Similarly, in the study of Chen et al., a relationship was found between the rs 11206510 minor $C$ allele and the risk of MI [39]. In the Mendelian randomization study of Schmidt et al. which included 550,000 individuals (50,000 of whom had diabetes), rs 11206510 rare $\mathrm{C}$ allele has been associated with higher fasting blood glucose, body mass index (BMI) and diabetes risk, although it was not found to be associated with low LDL-C levels and HbA1c levels [40]. Dysglycemia may be the result of lowering LDL-C. However, it was not yet known whether lowering LDL-C with PCSK9 inhibitors causes diabetes or not.

No difference was found between the patient and control groups in terms of TG, LDL-C and HDL-C in the study of Wang et al. [41], whereas in our study, LDL-C was lower and HDL-C was higher in OS-CAD group comparing with S-CAD group ( $p=0.042, p=0.008$, respectively). Qi et al. reported that the HbA1c levels and the percentages of hypertension and hypercholesterolemia in diabetic patients who developed CHD were higher than those who did not develop CHD [38]. In our study, the serum levels of HbA1c and the frequencies of hyperlipidemia and Type $2 \mathrm{DM}$ were found to be higher in S-CAD group compared to the OS-CAD group ( $p=0.015, p=0.001, p=0.007$, respectively).

Decreased EF percentages (\%) are one of the signs of CAD, and changes in left ventricular EF\% has been reported to be associated with the severity of CAD in previous studies [42,43]. In our study, a significant decrease was found in S-CAD group than OS-CAD group in terms of EF\% ( $p=0.007)$. Similar to our findings, Bal et al., reported that EF\% was found to be lower in patients with restenosis compared to patients without restenosis after stenting ( $p=0.023)$ [44].

In our study, the frequency of the E670G mutant G allele was found to be higher in S-CAD group than in OS-CAD ( $p=0.015)$. Besides, hyperlipidemia ( $<<0.001)$ and the PCSK9 E670G (rs505151) G allele ( $\mathrm{p}=0.02)$ were determined for the risk of restenosis between S-CAD and OS-CAD groups in the logistic regression analysis showing that the PCSK9 E670G (rs505151) G allele may be an effective risk factor in the development of restenosis. Slimani et al. reported that the frequency of the E670G-G allele in the Tunisian population was higher in CAD patients than controls $(0.132 \mathrm{vs}$. $0.068, \mathrm{p}=0.030)$. They also stated that plasma total-C and LDL-C levels were higher in $670 \mathrm{G}$ carriers than those without mutations (Total-C: $6.78 \mathrm{vs} .4 .92 \mathrm{mmol} / \mathrm{I}, \mathrm{p}<0.0001, \mathrm{LDL}-\mathrm{C}: 4.60 \mathrm{vs}$. $3.00 \mathrm{mmol} / \mathrm{I} \mathrm{p}=$ 0.001) [45]. Norata et al. suggested that the E670G-G allele is associated with high LDL-C and total-C [26]. In the meta-analysis study of Qiu et al., the E670G-G allele was found to be associated with high TG, LDL-C and cardiovascular disease risk [46]. Chen et al. suggested the association between E670G mutant allele and LDL-C and risk of atherosclerosis [47]. Similarly, in our study, LDL-C levels were found to be higher in E670G-G allele carriers compared to individuals with AA genotype in S-CAD group $(125.32 \pm 49.43$ vs. $107.90 \pm 34.18, p=0.036)$. Unlike our results and other studies suggesting the association between $E 670 G$ mutation and CAD $[45,46]$. Hsu et al. reported in their study with 202 patients with CAD and 614 controls in Taiwan-China population that E670G-G allele frequency was found to be similar in CAD and control groups ( $p>0.05)$ and LDL-C levels in E670G-G allele carriers were significantly low (2.78 \pm 0.82 mmol/L vs. $3.02 \pm 0.85 \mathrm{mmol} / \mathrm{L}, \mathrm{p}=0.029)$ [48]. In our study, borderline significance $(p=0.05)$ and lower TG levels were detected in E670G-G allele carriers of $0 S-C A D$ patients compared to individuals with AA genotype in the same group.

ApoE, another ligand of LDLR, is the apoprotein component of LDL and VLDL and is a polymorphic glycoprotein that acts as a ligand for chylomicron residue receptors [17]. Since ApoE2 contains cysteine instead of arginine in E3 and E4 at the 158th position, it was weakly bound to LDLR, causing an increase in VLDL levels in plasma. ApoE4 causes less gene expression (downregulation) of LDL receptors, leading to an increase in LDL levels in plasma. Most studies have found an association between $\mathrm{CHD}$ and the $A p o E 4$ allele [22, 23]. Xu et al. reported that the risk of CHD in the Caucasian population is high in ApoE4 carriers and low in ApoE2 carriers [49].

In the study conducted by Wang et al. with CHD patients in the Chinese population, it was reported that total-C, TG and LDL-C levels were higher in patients with ApoE4 allele compared to those who had homozygous ApoE3, and ApoE4 allele was associated with hypertriglyceridemia when ApoE3/E3 genotype was selected as reference in the multivariate logistic regression analysis [50]. In the study of Liu et al., it was reported that ApoE4 allele carriers have a 1.64 times higher risk of type $2 \mathrm{DM}$ and 1.80 times higher risk of cardiovascular disease in Chinese Hakka population [51]. In our study, the frequency of the ApoE epsilon polymorphism E3E4 genotype was found to be higher in the S-CAD group compared to the OS-CAD group (15.6\% vs $9.8 \%$ ), however, it was not statistically significant. In the study conducted by Atis et al. with CAD patients in the Turkish population, no significant difference was found between E2E2, E2E3, E2E4, E3E3, E3E4, E4E4 genotypes [52]. In our study, higher TG levels ( $p<0.001)$ were found in patients with the ApoE4 allele in the S-CAD group compared to the OSCAD group. There was no significant relationship between the ApoE4 allele and serum lipid parameters in the OS-CAD group. In the study conducted by Karahan et al. in the Turkish population, it was stated that CAD patients carrying ApoE4 allele had higher levels of LDL-C ( $p=0.001)$ and total-C ( $p=0.03)$

Page $9 / 13$ 
compared to those who did not [53]. In the study of Attila et al., it was shown that the ApoE4 allele was associated with the development of CAD in the Turkish population, but the effect of E2 and E4 alleles on lipid levels was not detected [54].

In our study, TG levels were found to be higher in individuals with ApoE2 allele in OS-CAD patients compared to those without E2 allele, but no significant difference was observed ( $p>0.05$ ). Serum VLDL-C levels in OS-CAD group were found to be significantly higher $(p=0.022)$ in patients with E2 allele compared to those without E2 allele. E2 allele was not found to be associated with serum lipid levels in S-CAD patients. Although serum TG levels were high in E2 allele carriers in S-CAD group, the difference was not statistically significant $(223.07 \pm 169.38$ vs. $167.97 \pm 11.46, p>0.05)$. In the study of Elmadbouh et al., hypercholesterolemia $(p=0.034)$ and high LDL-C $(p=0.003)$ were reported in patients carrying ApoE4 compared to CAD patients carrying ApoE3, and high TG levels $(\mathrm{p}=0.037)$ were reported in those carrying ApoE2 [55].

ApoER (LRP8) is an important molecule in lipoprotein metabolism. It functions in the removal of LDL-C and VLDL-C from plasma and has been associated with the risk of $\mathrm{Ml}$ in western societies. The $R 952 Q$ (rs5174) mutation converts arginine to glutamine. It has been associated with familial risk of CAD and premature MI in Caucasian populations [56]. In the study of Lieb et al. which was conducted with a German population, no association was observed between $R 952 Q$ mutation and familial MI or CAD [57]. In our study, just fasting blood glucose level was found to be higher in those with the ApoER-rs5174 [G> A (C> T)] $\mathrm{G}$ allele $(G G+G A)$ in OS-CAD patients compared to those with homozygous AA genotype $(p<0.001)$ in the same group.

In our study, a statistically significant difference was observed between the groups with and without the ApoE3 allele in the S-CAD group in terms of glucose and $\mathrm{HbA} 1 \mathrm{c}(\mathrm{p}<0.001$ and $\mathrm{p}=0.044$, respectively). In a study conducted with the Han Chinese population with individuals aged 70 and over, higher fasting blood glucose levels were found in individuals with the E3E3 genotype compared to individuals with the E2E2 and E4E4 genotypes ( $\mathrm{p}=0.047)$ and the ApoE3E3 genotype was found to be associated with diabetes [58]. In the study of El-Lebedy et al., diabetic patients with the E3E4 genotype showed a 2.4 fold increased risk of cardiovascular disease (95\% Cl: 1.14-5.19, $p=0.02)$ and E3E4 genotype was found as an independent risk factor for cardiovascular disease $(\mathrm{OR}=2.3, \mathrm{p}=0.009)$ but not for type $2 \mathrm{DM}(\mathrm{OR}=1.7, \mathrm{p}=0.28)$. However, the $E 4$ allele was reported as an independent risk factor for both type $2 \mathrm{DM}(\mathrm{OR}=2.2, \mathrm{p}=$ $0.04)$ and cardiovascular disease $(\mathrm{OR}=3.0, \mathrm{p}=0.018)$ and type $2 \mathrm{DM}$ patients with $E 4$ allele have 5.9 times increased risk of cardiovascular disease development [59].

In conclusion, while our findings revealed that PCSK9-rs505151 (E670G)- G allele and hyperlipidemia may be effective risk factors in the development of restenosis. Further studies on larger study group with expression of PCSK 9 gene and determination of the role of E670G substitution and in different ethnic groups are needed to confirm the association of this polymorphism with risk of restenosis.

\section{Declarations}

\section{Acknowledgement}

The present study was supported by the Istanbul University Scientific Research Projects Unit

(Project No: TSA-2018-30548 and TYO-2019-32124).

\section{Compliance with ethical standards}

\section{Conflict of interest}

Authors declare that no confict of interests exists.

\section{Ethical approval}

All procedures performed in this study were in accordance with the ethical standards of the institutional and/or national research committee and with the Declaration of Helsinki (2013). The study protocol was approved by the Local Ethical Commitee of Istanbul University, Faculty of Medicine (Protocol No:

2017/317, date: $7^{\text {th }}$ April, 2017)

\section{Informed consent}

Each individual in this study gave written informed consent prior to physical examination and blood sample collection.

\section{References}

1. Thanassoulis G, Ramachandran SV (2010) Contemporary Reviews in Cardiovascular Medicine, Genetic Cardiovascular Risk Prediction Will We Get There? Circulation 122, 2323-2334.

2. Williams KJ, Tabas I (1995) The response-to-retention hypothesis of early atherogenesis. Arteriosclerosis, Thrombosis, and Vascular Biology 15(5), 551562.

3. Mendis S, Nordet P, Fernadez-Britto JE, Sternby N (2005) Report for the Pathobiological Determinants of Atherosclerosis in Youth (PBDAY) Research Group: Atherosclerosis in children and young adults: An overview of the World Health Organization and International Society and Federation of Cardiology Study on Pathobiological Determinants of Atherosclerosis in Youth Study (1985-1995). Prevention and Control 1, 3-15.

4. Davis NE (2005) Atherosclerosis: An inflammatory process. Journal of Insurance Medicine 37, 72-75. 
5. Borissoff Jl, Heeneman S, Kilinç E, Kassák P, Van Oerle R, Winckers K, et al. (2010) Early atherosclerosis exhibits an enhanced procoagulant state. Circulation 122(8), 821-830.

6. Nordmann AJ, Hengstler P, Leimenstoll BM, Harr T, Young J, Bucher HC (2004) Clinical outcomes of stents versus balloon angioplasty in non-acute coronary artery disease. A meta-analysis of randomized controlled trials. Eur Heart J. 25(1), 69-80.

7. Indolfi C, Pavia M, Angelillo IF (2005) Drug-eluting stents versus bare metal stents in percutaneous coronary interventions (a meta-analysis). American Journal of Cardiology 95(10), 1146-1152.

8. Inoue T, Node K (2009) Molecular basis of restenosis and novel issues of drug-eluting stents. Circulation Journal 73(4), 615-621.

9. Abifadel M, Varret M, Rabes JP, Allard D, Ouguerram K, Devillers M, et al. (2003) Mutations in PCSK9 cause autosomal dominant hypercholesterolemia. Nat Genet. 34, 154156.

10. Sun XM, Eden ER, Tosi I, Neuwirth CK, Wile D, Naoumova RP, Soutar AK (2005) Evidence for effect of mutant PCSK9 on apolipoprotein B secretion as the cause of unusually severe dominant hypercholesterolaemia. Hum Mol Genet. 14, 1161-1169.

11. Hooper AJ, Marais AD, Tanyanyiwa DM, Burnett JR (2007) The C679X mutation in PCSK9 is present and lowers blood cholesterol in a Southern African population. Atherosclerosis 193(2), 445-448.

12. Akram ON, Bernier A, Petrides F, Wong G, Lambert G (2010) Beyond LDL Cholesterol, a New Role for PCSK9. Arterioscler Thromb Vasc Biol. 30, 12791281.

13. Poirier S, Mayer G, Poupon V, McPherson PS, Desjardins R, Ly K, et al. (2009) Dissection of the endogenous cellular pathways of PCSK9-induced LDL receptor degradation: evidence for an intracellular route. J Biol Chem. 284, 28856-28864.

14. Lagace TA (2014) PCSK9 and LDLR degradation: regulatory mechanisms in circulation and in cells. Curr Opin Lipidol 25(5), 387-393.

15. Duff CJ, Hooper NM (2011) PCSK9: an emerging target for treatment of hypercholesterolemia. Expert Opin Ther Targets 15(2), 157-168.

16. Park SW, Moon YA, Horton JD (2004) Posttranscriptional regulation of low density lipoprotein receptor protein by proprotein convertase subtilisin/kexin type 9a in mouse liver. J Biol Chem. 279, 50630-50638.

17. Mahley RW (1988) Apolipoprotein E: Cholesterol transport protein with expanding role in cell biology. Science 240, $622-630$.

18. Weisgraber KH, Rall SC, Mahley RW (1981) Human E apoprotein heterogeneity. Cysteine-Arginine interchanges in the amino acid sequence of the apo E isoforms. J Biol Chem. 256, 9077-9083.

19. Weisgraber KH, Innerarity TL, Mahley RW (1982) Abnormal lipoprotein receptor-binding activity of the human E apoprotein due to cysteine-arginine interchange atherosclerosis a single site. J Biol Chem. 357, 2518-2521.

20. Ehnholm C, Mahley RW, Chappel DA, Weisgraber KH, Ludwig E, Witztum L (1984) Role of apolipoprotein E in the lipolytic conversion of beta-very low density lipoproteins to low density lipoproteins in type III hyperlipoproteinemia. Proc Natl Acad Sci USA 81, 5566-5570.

21. Surguchov AP, Boerwinkle E, Sharett AR, Patsch WP (1994) Apolipoprotein E genotype and lipid transport: insight into the role of the epsilon4 allele. Atherosclerosis 106, 119-121.

22. Lenzen HJ, Assman G, Buckwalsky R, Schulte H (1986) Association of apolipoprotein E polymorphism, low density lipoprotein cholesterol, and coronary artery disease. Clin Chem. 32, 778-781.

23. Muros M, Rodriguez-Ferrer C (1996) Apolipoprotein E polymorphism influence on lipids, apolipoproteins and Lp (a) in a Spanish population underexpressing apo E4. Atherosclerosis 121, 13-21.

24. Pedersen JC, Berg K (1990) Gene-gene interaction between the low density lipoprotein receptor and apolipoprotein E loci affects lipid levels. Clin Genet. 38(4), 287-294.

25. Yılmaz-Aydogan H, Kucukhuseyin O, Kurnaz O, Akadam-Teker B, Kurt O, Tekeli A, Ozturk O, Isbir T (2012) Investigation of Polymorphic Variants of PPARD and APOE Genes in Turkish Coronary Heart Disease Patients. DNA Cell Biol. 31(5), 867-875.

26. Norata GD, Garlaschelli K, Grigore L, Raselli S, Tramontana S, Meneghetti F, et al. (2010) Effects of PCSK9 variants on common carotid artery intima media thickness and relation to ApoE alleles. Atherosclerosis 208, 177-182.

27. Ason B, van der Hoorn JW, Chan J, Lee E, Pieterman EJ, Nguyen KK, et al. (2014) PCSK9 inhibition fails to alter hepatic LDLR, circulating cholesterol, and atherosclerosis in the absence of ApoE. J Lipid Res. 55(11), 2370-2379.

28. Tavori H, Giunzioni I, Predazzi IM, Plubell D, Shivinsky A, Miles J, et al. (2016) Human PCSK9 promotes hepatic lipogenesis and atherosclerosis development via ApoE- and LDLR-mediated mechanisms. Cardiovasc Res. 110(2), 268-278.

29. Cohen J, Pertsemlidis A, Kotowski IK, Graham R, Garcia CK, Hobbs HH (2005) Low LDL cholesterol in individuals of African descent resulting from frequent nonsense mutations in PCSK9. Nat. Genet. 37, 161-165.

30. Berge KE, Ose L, Leren TP (2006). Missense mutations in the PCSK9 gene are associated with hypocholesterolemia and possibly increased response to statin therapy. Arterioscler. Thromb. Vasc. Biol. 26,1094-1100.

31. Timpson NJ, Najjar SS, Stringham HM, Strait J, Duren WL, Maschio A, et al. (2008) Newly identified loci that influence lipid concentrations and risk of coronary artery disease. Nat Genet. 40, 161-169.

32. Guella I, Asselta R, Ardissino D, Merlini PA, Peyvandi F, Kathiresan, et al. (2010) Effects of PCSK9 genetic variants on plasma LDL cholesterol levels and risk of premature myocardial infarction in the Italian population. J Lipid Res. 51, 3342-3349.

33. Postmus I, Trompet S, de Craen AJ, Buckley BM, Ford I, Stott DJ, et al. (2013) PCSK9 SNP rs11591147 is associated with low cholesterol levels but not with cognitive performance or noncardiovascular clinical events in an elderly population. Journal of lipid research 54(2), 561-566. 
34. Eroglu Z, Vardarli A, Duzgun Z, Gunduz C, Cetintas V, Kayıkcioglu M (2018) Case-control study on PCSK9 R496W (rs374603772) and D374Y (rs137852912) mutations in Turkish patients with primary dyslipidemia. Anatolian Journal of Cardiology 19, 334-340.

35. Kaya E, Kayıkçıoğlu M, Tetik Vardarlı A, Eroğlu Z, Payzın S, Can L (2017) PCSK 9 gain-of-function mutations (R496W and D374Y) and clinical cardiovascular characteristics in a cohort of Turkish patients with familial hypercholesterolemia. Anatolian Journal of Cardiology 18(4), 266-272.

36. Ferreira J, Xhaard C, Lamiral Z, Borges-Canha M, Neves J, et al. (2020) PCSK9 Protein and rs562556 Polymorphism Are Associated With Arterial Plaques in Healthy Middle-Aged Population: The STANISLAS Cohort. Journal of the American Heart Association, Wiley-Blackwell 9 (7), pp.e014758.

37. Zhang, L., Yuan, F., Liu, P., Fei, L., Huang, Y., Xu, L., et al. 2013. Association between PCSK9 and LDLR gene polymorphisms with coronary heart disease: Case-control study and meta-analysis. Clinical Biochemistry 46 (9), 727-732.

38. Qi, L., Parast, L., Cai, T., Powers, C., Gervino, E.V., Hauser, T.H., Hu, F.B., Doria, A. 2011. Genetic susceptibility to coronary heart disease in type 2 diabetes: 3 independent studies. Journal of the American College of Cardiology 58(25), 2675-2682.

39. Chen QF, Wang W, Huang F, Huang Z, Huang DL, Li T, et al. (2017) Relationship between rs11206510 and susceptibility, risk factors, and clinical characteristics of acute myocardial infarction in a Chinese Han population. Int J Clin Exp Med. 10(8), 12090-12100.

40. Schmidt A, Swerdlow D, Holmes M, Patel R, Fairhurst-Hunter Z, Lyall D, Hartwig F, et al. (2017) PCSK9 genetic variants and risk of type 2 diabetes: a mendelian randomisation study. The Lancet Diabetes and Endocrinology 5 (2), 97-105.

41. Wang S, Cheng ZY, Zhao ZN, Quan XQ, Wei Y, Xia DS, et al. (2016) Correlation of serum PCSK9 in CHD patients with the severity of coronary arterial lesions. European Review for Medical and Pharmacological Sciences 20, 1135-1139.

42. Dorbala S, Vangala D, Sampson U, Limaye A, Kwong R, Di Carli MF (2007) Value of vasodilator left ventricular ejection fraction reserve in evaluating the magnitude of myocardium at risk and the extent of angiographic coronary artery disease: a 82Rb PET/CT study. J Nucl Med. 48, 349-58.

43. Squeri A, Gaibazzi N, Reverberi C, Caracciolo MM, Ardissino D, Gherli T (2012) Ejection fraction change and coronary artery disease severity: a vasodilator contrast stress-echocardiography study. J Am Soc Echocardiogr. 25(4), 454-9.

44. Bal UA, Yıldıır A, Aydınalp A, Kaynar G, Kanyılmaz S, Koza M, Müderrisoğlu IH (2014) Could plasma asymmetric dimethylarginine level be a novel predictor beyond the classic predictors of stent restenosis? Anatol J Cardio. 14(6), 491-497.

45. Slimani A, Harira Y, Trabelsi I, Jomaa W, Maatouk F, Hamda KB, Slimane MN (2014) Effect of E670G Polymorphism in PCSK9 Gene on the Risk and Severity of Coronary Heart Disease and Ischemic Stroke in a Tunisian Cohort. J Mol Neurosci. 53(2), 150-7.

46. Qiu C, Zeng P, Li X, Zhang Z, Pan B, Peng ZYF.,et al. 2017. What is the impact of PCSK9 rs505151 and rs11591147 polymorphisms on serum lipids level and cardiovascular risk: a meta-analysis. Lipids Health Dis. 12, 16(1):111.

47. Chen SN, Ballantyne CM, Gotto AM Jr, Tan Y, Willerson JT, Marian AJ (2005) A common PCSK9 haplotype, encompassing the E670G coding single nucleotide polymorphism, is a novel genetic marker for plasma low-density lipoprotein cholesterol levels and severity of coronary atherosclerosis. $\mathrm{J}$ Am Coll Cardiol. 45(10), 1611-9.

48. Hsu LA, Teng MS, Ko YL, Chang CJ, Wu S, Wang CL, Hu CF (2009) The PCSK9 gene E670G polymorphism affects low-density lipoprotein cholesterol levels but is not a risk factor for coronary artery disease in ethnic Chinese in Taiwan. Clin Chem Lab Med. 47(2), 154-8.

49. Xu M, Zhao J, Zhang Y, Ma X, Dai Q, Zhi H, Wang B, Wang L (2016) Apolipoprotein E Gene Variants and Risk of Coronary Heart Disease: A Meta-Analysis. Biomed Res Int. 2016:3912175.

50. Wang C, Yan W, Wang H, Zhu J, Chen H (2019) APOE polymorphism is associated with blood lipid and serum uric acid metabolism in hypertension or coronary heart disease in a Chinese population. Pharmacogenomics 20(14), 1021-1031.

51. Liu S, Liu J, Weng R, Gu X, Zhong Z (2019) Apolipoprotein E gene polymorphism and the risk of cardiovascular disease and type 2 diabetes. BMC Cardiovasc Disord. 14, 19(1), 213.

52. Atis O, Sahin S, Ceyhan K, Ozyurt H, Akbas A, Benli I (2016) The Distribution of Apolipoprotein E Gene Polymorphism and Apolipoprotein E Levels among Coronary Artery Patients Compared to Controls. The Eurasian journal of medicine 48(2), 90-94.

53. Karahan Z, Ugurlu M, Ucaman B, Ulug AV, Kaya I, Cevik K, et al. (2015) Relation between Apolipoprotein E gene polymorphism and severity of coronary artery disease in acute myocardial infarction. Cardiol Res Pract. 2015, 363458.

54. Attila G, Acartürk E, Eskandari G, Akpinar O, Tuli A, Kanadaşi N, Kayrin L (2001) Effects of apolipoprotein E genotypes and other risk factors on the development of coronary artery disease in Southern Turkey. Clinica Chimica Acta 312(1-2), 191-196.

55. Elmadbouh I, Elghobashy Y, Abd-Allah E, Reda AA, Fathe A, Tayel S, Abd-Elhakim T (2013). Relationship of apolipoprotein E polymorphism with lipid profiles in atherosclerotic coronary artery disease. The Egyptian Heart Journal 65 (2), 71-78.

56. Shen, G.Q., Li, L., Girelli, D., Seidelmann, S.B., Rao, S., Fan, C., et al. 2007. An LRP8 variant is associated with familial and premature coronary artery disease and myocardial infarction. Am J Hum Genet. 81(4), 780-91.

57. Lieb W, Zeller T, Mangino M, Götz A, Braund P, Wenzel JJ. et al. (2008) Lack of association of genetic variants in the LRP8 gene with familial and sporadic myocardial infarction. J Mol Med (Berl) 86(10), 1163-70.

58. Ban CX, Zhong L, Wang T, Zhu MJ, Wang JH, et al. (2016) Enhanced Diabetes Susceptibility in Community Dwelling Han Elders Carrying the Apolipoprotein E 3/3 Genotype. PLOS ONE 11(3), e0151336.

59. El-Lebedy D, Raslan HM, Mohammed AM (2016) Apolipoprotein E gene polymorphism and risk of type 2 diabetes and cardiovascular disease. Cardiovascular diabetology 15,12

\section{Supplementary Files}

Page $12 / 13$ 
This is a list of supplementary files associated with this preprint. Click to download.

- Supplementalfile.doc

Page 13/13 